\title{
New Insight into Mechanisms of Allograft Transplantation in the Rat by Differential Display: Macrophage Scavenger Receptor-A Brings to Light
}

\author{
Elise Chiffoleau, Jean-Marie Heslan, Jean-Paul Soulillou, and Maria Cristina Cuturi \\ INSERM Unité 437 and Institut de Transplantation et de Recherche en \\ Transplantation (ITERT), Nantes, France \\ Accepted August 1, 2000.
}

\begin{abstract}
Background: Donor specific tolerance to heart allografts is induced in LEW.1A rat recipient by two donor LEW.1W blood transfusions prior engraftment. Although the tolerant allograft is infiltrated by leukocytes, graft infiltrating cells are only expressing low levels of the Th1- or Th2-related cytokines suggesting that induction of tolerance is an active phenomenon in which the mechanisms remain to be elucidated. Materials and Methods: Differential display (DD) method was applied on RNAs extracted from graft infiltrating cells (GIC) derived from allografts either from rejecting untreated rats or donor-specific blood transfusion treated tolerant rats. Quantitative RT/ PCR was performed to confirm mRNA expressions of the selected genes.

Results: Among the six differentially displayed DNAs (ddDNA) overexpressed in GIC from re-
\end{abstract}

jected allografts, the macrophage scavenger receptor-A (A:D 13265) was identified; it exhibited a stricking induction of mRNA expression from day 1 to 7 after transplantation. Among the seven ddDNAs overexpressed in GIC from tolerant allografts, the 3-hydroxy-3-methyl glutaryl coenzyme-A reductase (A:M29249) and an "unknown gene" (ddDNA EC9) were identified and both were confirmed to be up-regulated by quantitative RT/PCR.

Conclusions: The relevance of these genes in transplantation has not yet been reported and must therefore be elucidated; they represent possible targets for immunointervention. Nevertheless, our data demonstrate that the DD is a powerful tool to identify new genes involved in organ transplantation.

\section{Introduction}

Transplantation is a common treatment for many vital organ end-stage diseases. Despite recent progress in immunointervention, acute or chronic rejection still occurs due to differences in the major histocompatibility complex (MHC) between the organ donor and the recipient. Therefore, the understanding of the immunological mechanisms leading to rejection or long-term tolerance to the graft remains a major goal to discover novel therapeutic targets. Thus, to identify new genes involved in allograft transplantation we have applied the

Correspondence and reprint requests to: Maria Cristina Cuturi, INSERM U437, 30 boulevard Jean Monnet, 44093 Nantes Cedex 93, France. E-mail: ccuturi@nantes.inserm.fr differential display (DD) method to our welldefined heart allograft model in the Lewis rat. Donor-specific tolerance to heart allografts was induced in LEW.1A recipient by two donorspecific LEW.1W blood transfusions (DST) prior to engraftment (1). The strain specificity of this tolerance was demonstrated by the acceptance of a donor-type skin graft whereas a third-party skin graft was rejected (2). Although the tolerant allograft was infiltrated by leukocytes, graft infiltrating cells (GIC) are only expressing low levels of the Th1 or Th2 related cytokines but produced high amounts of the active form of TGF- $\beta$, which could exert its immunosuppressive effect in this model (3). Moreover, tolerant animals failed to mount an efficient anti-donor humoral response whereas the transfer of sera from rejecting animals 
triggered rejection in tolerant animals (4). Taken together, these data suggest that such tolerance is an active phenomenon in which mechanisms are not yet identified.

By comparing the DD profiles of RNAs from GIC isolated either from rejected allografts or tolerant allografts, we identified 13 cDNAs differentially expressed between both these situations. Regarding their involvement in immunological processes, we have selected two genes, and an "unknown gene" and followed their mRNAs expression levels from day 1 to 7 after transplantation by real-time quantitative RT/PCR. The ddDNA EC14 corresponding to the macrophage scavenger receptor-A (MSR-A) was clearly overexpressed in GIC from rejected compared to tolerant grafts and by quantitative RT/PCR; it exhibited up to 25-fold induction in mRNA expression on day 1 after transplantation. Conversely, the 3-hydroxy-3-methylglutaryl coenzyme A reductase (HMGCoAred) (ddDNA EC7) and an "unknown gene" (ddDNA EC9), were upregulated in tolerant allografts. Our results showed that these molecules are likely to be involved in the specific control of the organ acceptance or rejection.

\section{Materials and Methods}

Animals

Eight- to 12-week-old adult male Lewis rats, from the LEW.IW (RTlu) and LEW.lA (RTla) inbred strains, were purchased from the Centre d'Elevage Janvier (Le Genest-Saint-Isle, France) and raised in conventional animal facilities.

\section{Transfusion and Transplantation}

The LEW.IW strain served as blood and heart donors, and the LEW.1A strain as recipient. To induce tolerance, $1 \mathrm{ml}$ of heparinized blood collected by cardiac puncture was IV injected into recipients 14 and 7 days before transplantation. Heterotopic heart engraftments were performed according to Ono and Lindsey (5). Graft function was evaluated daily by palpitation measurement through the abdominal wall. LEW.1W heart allografts in untreated LEW.1A recipients were rejected in $6.6 \pm 0.6$ days, whereas they survived over 100 days in DSTtreated LEW.1A recipients (1). LEW.1A syngeneic grafts were also performed as controls.

\section{Graft Infiltrating Cell Extraction}

Heart allografts removed on day 5 were finely minced in cold phosphate-buffered saline (PBS), and gently pressed through a stainless steel mesh. Graft-infiltrating cells were isolated using density gradient centrifugation on Ficoll (Amersham Pharmacia Biotech, Sweden), and finally washed twice.

\section{RNA Extraction}

Total RNAs from whole allografts were extracted in guanidium-isothiocyanate solution followed by cesium chloride ultracentrifugation (6). Total RNAs from GIC were extracted as described by Chomczynski and Sacchi (7). All RNAs were quantified by UV absorbance at $260 \mathrm{~nm}$.

\section{Differential Display}

Anchored oligo-dT primers: $\mathrm{HT}_{11} \mathrm{~A}, \mathrm{HT}_{11} \mathrm{G}$, $\mathrm{HT}_{11} \mathrm{C}$, and random 13 mers and 10 mers oligonucleotide primers were synthesized by Genosys (Cambridge, UK). Total RNAs from GIC were treated with RNase-free DNase (1U/ $\mu l$, Boehringer, Germany) to eliminate genomic DNA. Two hundred ng of RNA were reverse transcribed with M-MLV reverse transcriptase (200 U/ $\mu \mathrm{l}$, Gibco Brl, UK) and one of the oligo (dT) primer $\mathrm{HT}_{11} \mathrm{~A}, \mathrm{HT}_{11} \mathrm{C}$, and $\mathrm{HT}_{11} \mathrm{G}$ $(0.2 \mu \mathrm{M})$ in $20 \mu \mathrm{l}$ reaction volume. Ten pg cDNA were then amplified by PCR in the presence of $0.1 \mu \mathrm{l}$ of $\alpha^{33} \mathrm{P} \mathrm{d}(\mathrm{ATP})(2,000 \mathrm{Ci} / \mathrm{mmol})$ and $180 \mathrm{nM}$ of arbitrary 10 or 13 mers as forward primers and $180 \mathrm{nM}$ of $\mathrm{HT}_{11} \mathrm{~A}, \mathrm{HT}_{11} \mathrm{C}$, or $\mathrm{HT}_{11} \mathrm{G}$ as reverse primers in the PerkinElmer 9600 thermocycler (PE Biosystem, Foster City, CA, USA) for 40 cycles consisting in denaturation at $94^{\circ} \mathrm{C}$ for $15 \mathrm{sec}$, annealing at $40^{\circ} \mathrm{C}$ for $1 \mathrm{~min}$, and extension at $72^{\circ} \mathrm{C}$ for 20 sec. Radiolabeled PCR products were run on $8 \mathrm{M}$ urea- $6 \%$ polyacrylamide gels that were dried and exposed to Xomat film (Kodak, Rochester, NY, USA) for autoradiography.

\section{Recovery and Reamplification of DNA Probes}

Differentially displayed bands were carefully located and cut out of the dried gel. The gel slice along with the $3 \mathrm{~mm}$ paper was rehydrated in $100 \mu \mathrm{l} \mathrm{H}_{2} \mathrm{O}$ for $10 \mathrm{~min}$. The ddDNAs were extracted by boiling for $15 \mathrm{~min}$ followed by ethanol precipitation in the presence of $0.3 \mathrm{M}$ $\mathrm{NaOAc}$ and $50 \mu \mathrm{g}$ of glycogen. Finally, the pellet 
was dissolved in $10 \mu \mathrm{l} \mathrm{H} \mathrm{H}_{2} \mathrm{O}$. Four microliters of eluted ddDNA were amplified in a $40 \mu \mathrm{l} \mathrm{PCR}$ reaction using the same primer set and conditions as used in the DD except that $20 \mu \mathrm{M}$ dNTP were used instead of $4 \mu \mathrm{M}$ and no radioactive isotope was added.

\section{Cloning and Sequencing of ddDNAs}

Reamplified ddDNAs were inserted into the PCRII vector using the TA cloning system (Invitrogen, Netherlands), then transformed in TOP10 bacteria and cloned on ampicilin-agar dishes. Cloned ddDNAs were sequenced with M13 primers annealing into the vector. Sequence homology searches were done by Blastn against the gene banks of the NCBI (National Center for Biotechnology) Internet site.

\section{Quantitative RT/PCR}

Quantitative RT/PCRs were performed on the ABI Prism 7700 (PE-Biosystems) using the TaqMan chemistry (PE-Biosystems under license of Roche Molecular Systems, Inc). This TaqMan system performed real-time kinetic PCR and true quantitative gene analysis (8). The sequences of gene specific primers are given in Table 1. Standards were prepared by PCR amplification of each target sequence using these primers and cDNAs derived from whole graft total RNAs; PCR products were extracted and the OD260 allowed the quantification of the template in the standards. The standards were diluted to load $10^{7}-10^{2}$ copies per well.

Total RNAs from grafts were extracted and reverse-transcribed using an oligo $\mathrm{dT}$ as previously described (3). A constant amount of cDNA corresponding to the reverse transcription of $100 \mathrm{ng}$ of total RNAs, or each dilution of the standard, were amplified using SYBR Green PCR Core Kit (PE Biosystems) containing $300 \mathrm{nM}$ of each primers in a final volume of $25 \mu \mathrm{l}$. Amplification reaction started by a 2-min step at $55^{\circ} \mathrm{C}$, then a 10 -min step at $95^{\circ} \mathrm{C}$, followed by 40 cycles at $95^{\circ} \mathrm{C}$ for $15 \mathrm{sec}, 60^{\circ} \mathrm{C}$ for $1 \mathrm{~min}$ for the housekeeping gene HPRT and unknown ddDNA EC9, or $55^{\circ} \mathrm{C}$ for MSR-A and $62^{\circ} \mathrm{C}$ for HMG CoAred. The PCR efficiency of all the standards were greater than $99 \%$, and the correlation index between the input copy numbers and the fluorescence were always greater than 0.95. Data were expressed as ratios of the number of copies of the specific gene to the number of copies of the HPRT gene.

\section{Statistical Analysis}

Statistical evaluation was performed using student's $t$-test for unpaired data and was considered significant if $p$-values were $<.05$. Data were expressed as mean \pm SEM.

\section{Results \\ Differential Display and Gene Identification}

Preliminary DD experiments with RNAs from whole grafts had produced virtually no differential patterns due to the large amount of RNAs from cardiac tissue. Thereafter in this work, we have used RNAs extracted from GIC isolated either from rejected allografts $(n=4)$, or tolerant allografts $(n=4)$. Total RNAs were processed according to DD procedure using 36 primer combinations. Experimental conditions were improved until results were reproducible and even loadings on the gels were judged according to the intensity of the several major conserved bands present in all the sample lanes. Displaying both series of samples side by side on gels, allowed discrimination of differences due to individuals and hence to select bands clearly modulated on at least three out of the four samples in the given series.

Table 1. Sequences of primers used for quantitative RT/PCR

$\begin{array}{ll}\text { Forward HPRT } & \text { 5'-GCGAAAGTGGAAAAGCCAAGT-3' } \\ \text { Reverse HPRT } & \text { 5'-GCCACATCAACAGGACTCTTGTAG-3' } \\ \text { Forward MSR-A } & \text { 5'-GGTGTTAAAGGTGATCGG-3' } \\ \text { Reverse MSR-A } & \text { 5'-GGTGTTAAAGGTGATCGG-3' } \\ \text { Forward HMGCoAred } & \text { 5'-GAAGTCAGCCTACAGAGCCA-3' } \\ \text { Reverse HMGCoAred } & \text { 5'-AGGAGCCCATAAATGATTCAG-3' } \\ \text { Forward ddDNA EC9 } & \text { 5'-CGAGGGAGTAGGGTCAGGT-3' } \\ \text { Reverse ddDNA EC9 } & \text { 5'-ATCGCAAGTTCCTGTTCCTGA-3' }\end{array}$


Figure 1 shows an enlargment of autoradiograms with differentially expressed bands of two representative ddDNAs: ddDNA EC14 overexpressed in the sample from rejected allografts (Fig. 1A) and ddDNA EC9, overexpressed in the samples from tolerant allografts (Fig. 1B).

The ddDNA bands were cut out of the acrylamide gel, PCR-amplified with the same pair of primers, then cloned in TopoA vector plasmid to be sequenced using the M13 primers. The ddDNA sequences were compared to those of the NCBI gene banks (National Center for Biotechnology) using blastn software for homology search. Out of the six ddDNAs overexpressed in GIC from rejected allografts, ddDNA EC6 and EC11 had 99\% homology from bases 173-269 with the Mss4 guanine nucleotide exchange factor of Rattus norvegicus (A: L10336). EC21 had 94\% homology with the $5^{\prime}$ end of the Mus musculus Fibroblast Growth Factor inducible gene (bases 388-495) (A: 42385). EC14 had $81 \%$ homology with the $3^{\prime}$ untranslated end of the human macrophage scavenger receptor-A (bases 1369-1405) (A: D 13265). Both remaining EC17 and EC 18 had
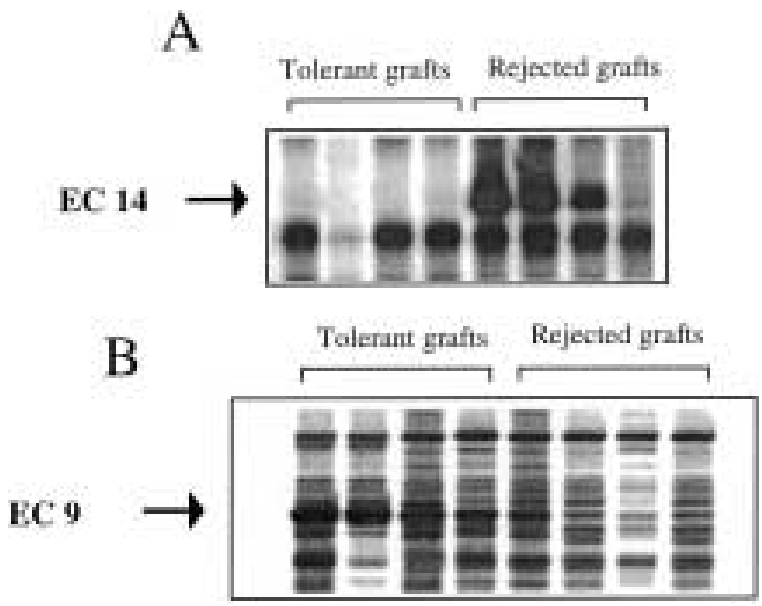

Fig. 1. Differential Display (DD) patterns comparing RNAs from graft infiltrating cells. (GIC) from rejected allografts $(n=4)$ and tolerant allografts $(n=4)$. Total RNAs extracted from GIC isolated from grafts removed on day 5 after transplantation were subjected to DD method using HT11G and primer 3 for ECl4 and HT11C and primer 7 for EC9. Autoradiograms of the $\alpha{ }^{33} \mathrm{P}$ dATP-labeled PCR products runned on $6 \%$ PAGE: Arrows show (A) ddDNA EC14 expressed in rejected grafts and (B) ddDNA EC9 overexpressed in tolerant grafts. no homology with any known genes or EST from the banks and so-called "unknown genes."

Among the seven ddDNAs overexpressed in GIC from tolerant allografts, four ddDNAs were homologous to known genes of the Rattus norvegicus: EC20 had 100\% homology over the 97 nucleotides of the $5^{\prime}$ end of nonmuscle myosin alkali light chain (bases 2-99) (A: S77858). EC5 and EC16 both exhibited 99\% homology with mitochondrial proteins of the 3' end of the ATPase subunit 6 (bases 586-828) (A: X14848) and to the $3^{\prime}$ end of the cytochrome C oxidase subunit 3 (bases 4362-4492) (A: V01574), respectively. EC7 had $95 \%$ homology with the 3' end of 3-hydroxy-3-methyl glutaryl Coenzyme-A reductase (bases 3739-3934) (A: M29249). Finally, EC19 had 86\% homology with the 3' end of a human nucleotide-bindingprotein mRNA (bases 886-960) (A: V01833) and also two "unknown genes" EC9 and EC10.

Surprisingly, although the DD method should produce DNAs from the $3^{\prime}$ end of the genes, some of our ddDNAs are located within the ORF or the 5' extremity of the genes.

We started to study the mRNA expression of MSR-A and HMGCoAred genes according to their reported functions in immunological pathways, and the "unknown gene" EC9. We decided not to further analyze the ddDNAs corresponding to genes with no obvious involvement in known immunological processes.

\section{Quantitative RT/PCR}

mRNA expression of the three selected genes were analyzed by real-time quantitative RT/ PCR in syngeneic grafts $(n=3)$ or in rejected $(n=3)$ or tolerant allografts $(n=3)$ removed on day $1,3,5$, or 7 after transplantation. Because in syngeneic grafts, there was only a mild cell infiltration, these samples served as baseline expression in absence of GIC. Specific primers were designed within open reading frames of MSR-A and HMGCoAred sequences found in the NCBI banks; they amplify uniquely target sequences and meet the TaqMan PCR requirements.

The ddDNA EC14 fragment exhibiting $81 \%$ homology with the 3' untranslated end of the human MSR-A was overexpressed in GIC of rejected allografts (Fig. 1A). The MSR-A mRNAs were consistently overexpressed (25fold on day $1(p<0.05), 3.7$-fold on day 5 , and 24-fold on day 7) in rejected allografts as 
compared to tolerant allografts by quantitative RT/PCR (Fig. 2A). In syngeneic grafts, MSRA expression was very low, suggesting that this molecule could be expressed by the GIC themselves as soon as day 1 after transplantation (Fig. 2A).

On DD gels, the HMGCoAred gene was slightly more expressed on day 5 after transplantation in GIC derived from tolerant allografts as compared to rejected ones (data not shown). On days 1 and 7, tolerant allografts ex- pressed significantly higher amounts of HMGCoAred than syngeneic grafts or rejected allografts ( 2 - and 4 -fold $(p<.05)$, respectively) by quantitative RT/PCR (Fig. 2B). In contrast, on day 5, rejected allografts expressed higher amounts than tolerant ones $(1.5$-fold $(p<.05))$. For HMGCoAred expression, there was a discrepancy between quantitative RT/PCR and the DD pattern exhibited on day 5 after transplantation. This discrepancy could be due to the difference in the materials used to extract
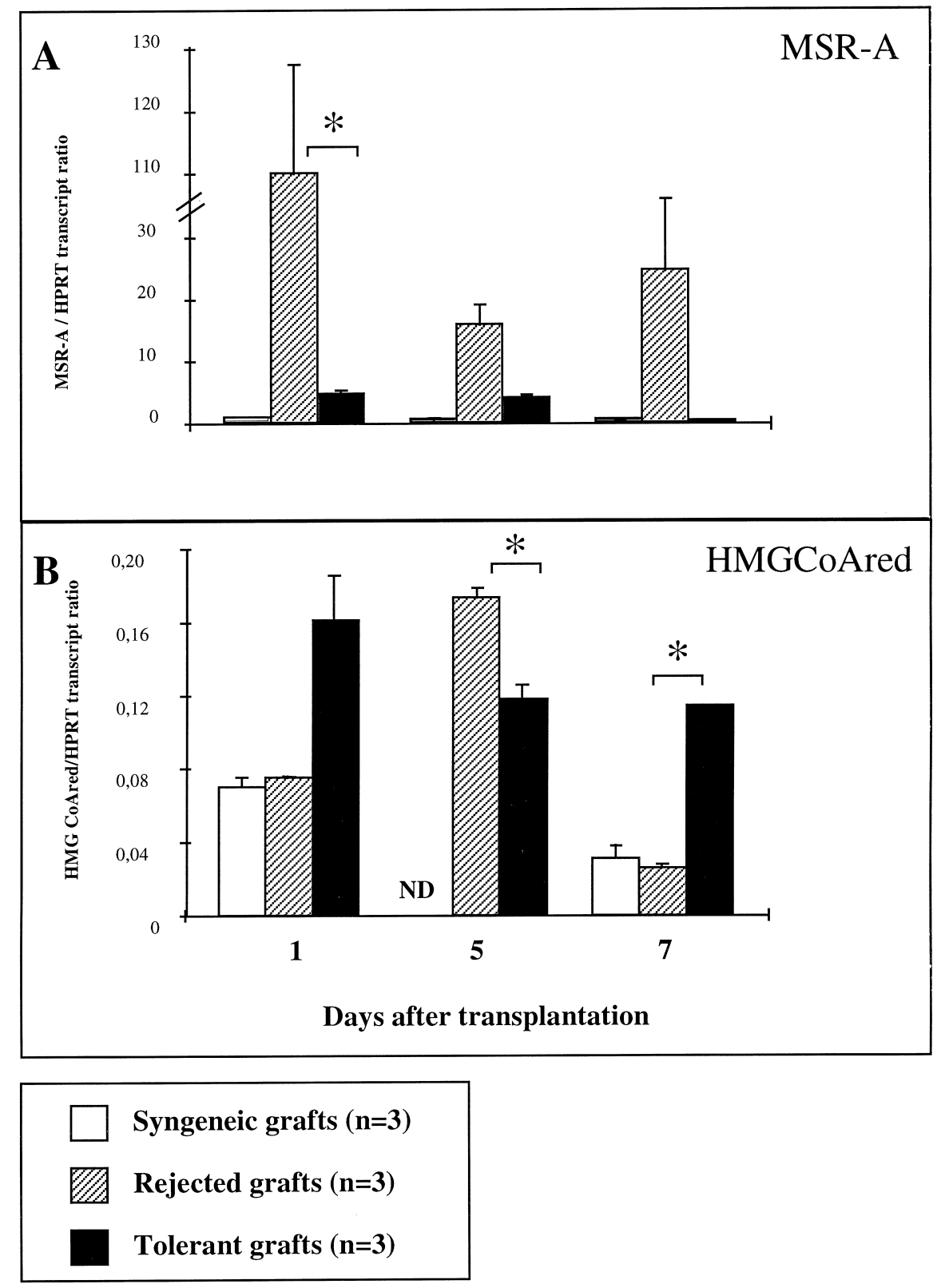

Fig. 2. Real-time quantitative RT/PCR of the selected genes. Quantification was performed on syngeneic grafts $(n=3)$, rejected allogeneic grafts $(n=3)$ and tolerant allogeneic grafts $(n=3) 1,5$, and 7 days after transplantation for (A) MSR-A and (B) HMG CoAred. Gene-specific transcripts were normalized to HPRT transcripts. ${ }^{*} p<0.05$. 
RNAs (i.e., solely GIC for the DD and whole allografts for the RT/PCR). Eventually, cardiac tissue cells which could express the HMGCoAred, could mask the up-regulation due to GIC in the tolerant allografts on day 5 after transplantation.

The "unknown gene" corresponding to ddDNA EC9 was overexpressed in GIC derived from tolerant allografts on DD gel (Fig. 1B). Specific primers were designed from the DD cloned sequence and used for quantitative RT/PCR. On day 1 and 3, tolerant and rejected grafts expressed similar amounts of ddDNA EC9 whereas on day 5 and 7, tolerant grafts expressed 3- and 4-fold more ddDNA EC9 mRNAs, respectively $(p<.05)$ (Fig. 3). An attempt to clone the full-length cDNA is underway.

\section{Discussion}

The DD patterns of amplified genes from GIC iolated from DST-treated tolerant recipients and untreated rejecting recipients exposed to the same surgical procedure were studied. We identified 13 ddDNAs differentially expressed during rejection or induction of tolerance. These ddDNAs were reamplified by PCR, cloned in a plasmid vector, sequenced, and the sequences were compared to those of the GenBank. We started to analyze three ddDNAs, two according to their immunological functions and one "unknown gene." Among the ten others, many were genes coding for cellular machinery proteins such as mitochondrial proteins or proteins involved in the intracellular trafficking. For the three selected genes, we analyzed their kinetics of expression on whole graft RNAs by quantitative RT/PCR.

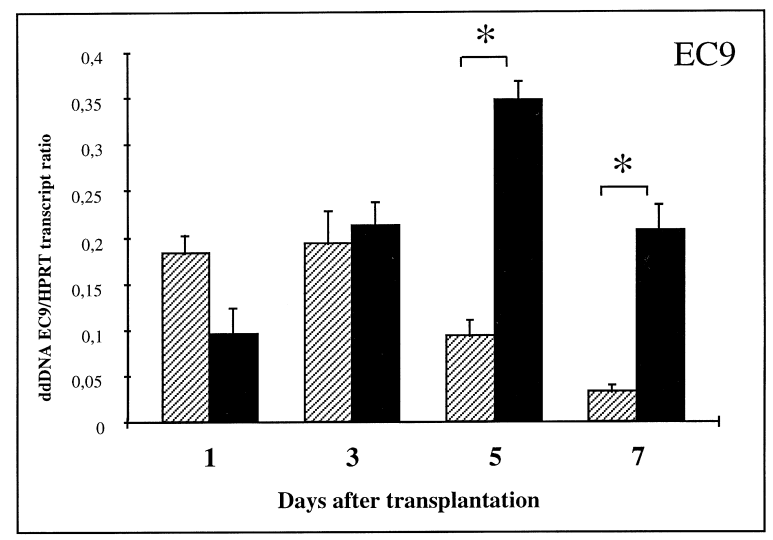

As soon as day 1 and at least during the first week after grafting, mRNA expression of MSR-A was strikingly up-regulated in rejected grafts. Such an involvement of MSR-A in transplantation has not been yet reported. MSR-As are trimeric glycoproteins expressed by macrophages that have been extensively described in the pathologic deposition of cholesterol in arterial walls during atherogenesis through the uptake of modified low density lipoproteins (LDL) (9). MSR-As, which recognize a wide range of polyanionic ligands including lipopolysaccharide, are overexpressed in activated macrophages and have a protective role in host defense (10).

After grafting, monocytes/macrophages represent more than $50 \%$ of total cell infiltration in rejected grafts (data not shown). Macrophages play an early role in cell-mediated immunity and their activation leads to expression of proinflammatory cytokines and recruitment of LT and NK $(11,12)$. In addition, the presence of activated macrophages in the graft has a predictive value for chronic or acute rejection (13). During rejection, macrophages rapidly migrate close to the endothelium where activated endothelial cells express adhesion receptors for monocytes such as VCAM-1 (vascular cell adhesion molecule 1), and secrete chemokines, which in turn stimulate monocytes to adhere to and migrate into the subendothelial space. It has been shown that in vivo, MSR-A functions as a receptor for macrophage adhesion in lymphoid and nonlymphoid organs. It has been suggested that MSR-A in combination with other macrophage adhesion molecules could contribute to signals for tissue-specific localization of mononuclear phagocytes in normal and pathologic states such as inflammation (14). Moreover, binding of ligands to MSR was

Rejected grafts $(n=3)$

Tolerant grafts $(n=3)$

Fig. 3. Real-time quantitative RT/PCR of the "unknown gene" EC9. Quantification was performed on rejected allogeneic grafts $(n=3)$ and tolerant allogeneic grafts $(n=3) 1,3,5$, and 7 days after transplantation. Gene-specific transcripts were normalized to HPRT transcripts. ${ }^{*} p<0.05$. 
shown to induce protein tyrosine phosphorylation and increased protein kinase $\mathrm{C}$, phospholipase $\mathrm{C}$ gammal, and phosphatidyl inositol-3$\mathrm{OH}$-kinase. This intracellular activation leads to the increase of urokinase-type plasminogen activator expression (15), induction of NF- $\kappa \mathrm{B}$, and transcription of TNF- $\alpha, \mathrm{NO}$, and ILl $(16,17)$.

Recent studies indicate that MSR-A does not merely act by scavenging modified proteins and particles for intracellular degradation but also by presenting such modified compounds as antigens to the immune system (18). It has also been shown that conversion of proteins to scavenger receptor ligands by maleylation increases their immunogenicity and leads to increased up-regulation of IFN $\gamma$ and down-regulation of IL4 and IL10 by maleyl-antigen immune spleen cells. MSR binding does not change the surface levels of costimulatory or adhesive molecules but higher levels of peptide-CMH complexes are expressed on spleen cells. Administration of maleylated antigens leads to the presentation of higher levels of stimulatory peptide-MHC complexes than injection of native antigens and tends to generate a relatively greater dominance of the Thl response (19). Therefore, in our model, we suggest that MSR-A could be involved in the indirect presentation of the donor specific antigens presented by activated macrophages to induce activation and expansion of specific T-cell clones.

Our results show an early and strong upregulation of the MSR-A mRNA expression during allograft rejection. The importance of its regulation is strengthened by the low expression of MSR-A during induction of tolerance, although the macrophage infiltrates are identical in both rejected and tolerant grafts (3). Moreover, it has been shown that MSR-A mRNA expression was inhibited by TGF $\beta$, a cytokine which we have reported to be overexpressed in our model of tolerance induction (3). In contrast, MSR-A expression is increased by ILl and IFN $\gamma(20)$, both of which are expressed during allograft rejection (3). This regulation pattern suggests that this molecule may provide a possible target for immunointervention in transplantation. Blockade of these receptors following organ transplantation may be of value in the prolongation of graft survival in combination with an anti-T-cell strategy.

Quantitative RT/PCR confirmed the overexpression of HMGCoAred in tolerant grafts compared to rejected grafts but, on days 1 and
7 only, and not on day 5. HMGCoAred converts HMGCoA to mevalonate, which is involved in the synthesis of cholesterol and other nonsterol by-products. The statins, inhibitors of this enzyme, are largely used in hypercholesterolemia to reduce plasma cholesterol levels in patients but also to prevent acute graft rejection (21). Imagawa et al. and Katznelson et al. have shown that besides its effect on chronic rejection, pravastatin could decrease the incidence of both acute rejection episodes and natural killer cell cytotoxicity after heart transplantation $(21,22)$. Lovastatin inhibits the in vitro proliferation of mitogen-stimulated T-lymphocytes and natural killer cell cytotoxicity. However, the patterns of HMGCoAred regulation does not suggest a role in acute rejection. This is reinforced by the observation that in the same LEW.1W to LEW.1A combination, HMGCoAred inhibitors does not influence graft survival (Soulillou et al., unpublished). However, this enzyme, which is up-regulated in allograft tolerance, might be expressed by or for a specific subset of cells involved in the induction of tolerance.

The "unknown gene" ddDNA EC9 was upregulated in tolerant grafts compared to rejected grafts. EC9 expression during the first week after transplantation shows that although on days 1 and 3, EC9 was similarly expressed by both tolerant and rejecting recipients, on days 5 and 7, its expression is up-regulated in the tolerant group whereas it is downregulated in the rejecting group. Therefore, the absence of this "unknown gene" is associated with the rejection phase. The EC9 function might be of interest because its expression could prevent the onset of the rejection. Fulllength cDNA cloning is in progress and could enable us to define the exact role of this gene in the process of induction of the tolerance.

Finally, the DD method had allowed us to identify genes not yet related to the transplantation, DD is therefore a convenient method to study a complex and multifactorial system like organ tranplantation. The exact role of the genes we have identified must be elucidated because they may serve as targets for immunointervention or markers in transplantation.

\section{References}

1. Soulillou JP, Blandin F, Gunther E, Lemoine V. (1984) Genetics of the blood transfusion effect on heart allografts in rats. Transplantation 38: 63-67. 
2. Josien R, Heslan M, Brouard S, Soulillou JP, Cuturi MC. (1998) Critical requirement for graft passenger leukocytes in allograft tolerance induced by donor blood transfusion. Blood 92: 4539-4544.

3. Josien R, Pannetier C, Douillard P, et al. (1995) Graft-infiltrating $\mathrm{T}$ helper cells, CD45RC phenotype, and Th1/Th2- related cytokines in donorspecific transfusion-induced tolerance in adult rats. Transplantation 60: 1131-1139.

4. Cuturi MC, Josien R, Cantarovich D, et al. (1994) Decreased anti-donor major histocompatibility complex class I and increased class II alloantibody response in allograft tolerance in adult rats. Eur. J. Immunol. 24: 1627-1631.

5. Ono K, Lindsey ES, (1969) Improved technique of heart transplantation in rats. J. Thorac. Cardiovasc. Surg. 57: 225-229.

6. Chirgwin JM, Przybyla AE, MacDonald RJ, Rutter WJ. (1979) Isolation of biologically active ribonucleic acid from sources enriched in ribonuclease. Biochemistry 18: 5294-5299.

7. Chomczynski, P, Sacchi N, (1987) Single-step method of RNA isolation by acid guanidinium thiocyanate-phenol-chloroform extraction. Anal. Biochem. 162: 156-159.

8. Yasuda T, Takeshita H, Nakazato E, Nakajima T, Hosomi O, Nakashima Y, Kishi K. (1998) Activity measurement for deoxyribonucleases I and II with picogram sensitivity based on DNA/SYBR Green I fluorescence. Anal. Biochem. 255: 274-276.

9. Ross R. (1993) The pathogenesis of atherosclerosis: a perspective for the 1990s. Nature 362: 801809.

10. Suzuki H, Kurihara Y, Takeya M, et al. (1997) A role for macrophage scavenger receptors in atherosclerosis and susceptibility to infection. Nature 386: 292-296.

11. MacPherson GG, Christmas SE. (1984) The role of the macrophage in cardiac allograft rejection in the rat. Immunol. Rev. 77: 143-166.

12. Han DJ, Kim SC, Lee HM, We YM, Kang HY, Yu ES. (1998) Implication of monocyte/macrophage infiltration into the graft following heart transplantation in rats. Transplant. Proc. 30: 3407.

13. Bogman MJ, Dooper IM, van de Winkel JG, et al. (1989) Diagnosis of renal allograft rejection by macrophage immunostaining with a CD14 monoclonal antibody, WT14. Lancet 2: 235-238.

14. Hughes DA, Fraser IP, Gordon S. (1995) Murine macrophage scavenger receptor: in vivo expression and function as receptor for macrophage adhesion in lymphoid and non-lymphoid organs. Eur. J. Immunol. 25: 466-473.

15. Hsu HY, Hajjar DP, Khan KM, Falcone DJ. (1998) Ligand binding to macrophage scavenger receptor-A induces urokinase-type plasminogen activator expression by a protein kinase-dependent signaling pathway. J. Biol. Chem. 273: 1240-1246.

16. Misra UK, Shackelford RE, Florine-Casteel K, et al. (1996) Maleylated-BSA induces hydrolysis of PIP2, fluxes of Ca2+, NF-kappaB binding, and transcription of the TNF-alpha gene in murine macrophages. J. Leuk. Biol. 60: 784-792.

17. Hamilton TA, Gainey PV, Adams DO. (1987) Maleylated-BSA suppresses IFN-gamma-mediated Ia expression in murine peritoneal macrophages. J. Immunol. 138: 4063-4068.

18. Nicoletti A, Caligiuri G, Tornberg I, Kodama T, Stemme S, Hansson GK. (1999) The macrophage scavenger receptor type A directs modified proteins to antigen presentation. Eur. J. Immunol. 29: 512-521.

19. Singh N, Bhatia S, Abraham R, et al. (1998) Modulation of $\mathrm{T}$ cell cytokine profiles and peptide-MHC complex availability in vivo by delivery to scavenger receptors via antigen maleylation. J. Immunol. 160: 4869-4880.

20. Grewal RP, Yoshida T, Finch CE, Morgan TE. (1997) Scavenger receptor mRNAs in rat brain microglia are induced by kainic acid lesioning and by cytokines. Neuroreport 8: 1077-1081.

21. Katznelson S, Wilkinson AH, Kobashigawa JA, et al. (1996) The effect of pravastatin on acute rejection after kidney transplantation-a pilot study. Transplantation 61: 1469-1474.

22. Imagawa DK, Dawson SR, Holt CD, et al. (1996) Hyperlipidemia after liver transplantation: natural history and treatment with the hydroxy-methylglutaryl-coenzyme A reductase inhibitor pravastatin. Transplantation 62: 934-942. 